\title{
Produtividade da batata em função da irrigação e do controle químico da requeima ${ }^{1}$
}

\author{
Edenir L. G rimm², Arno B. H eldwein'2, Sidinei Z. Radons², Ivan C. Maldaner², \\ G ustavo Trentin $^{3}$ \& Leosane C. Bosco ${ }^{2}$
}

\begin{abstract}
RESU MO
O bjetivou-se com esse trabalho determinar o efeito de diferentes níveis de irrigação e controle químico na produtividade e ocorrência de requeima na cultura da batata, cultivar "Asterix", em Santa M aria, RS. O s experimentos foram realizados no Departamento de Fitotecnia da U niversidade Federal de Santa Maria, o primeiro no verão de 2005/2006 e o segundo no outono de 2006. O delineamento experimental utilizado foi o de blocos ao acaso, com quatro repetições. U tilizou-se o sistema Blitecast para determinar o momento da aplicação de fungicidas, através do acúmulo de valores de severidade (VS). A irrigação foi realizada por aspersão, utilizando-se a dotação de 1,0 ETm, 0,75 ETm, 0,50 ETm e sem irrigação (Test). Os resultados mostraram que em períodos relativamente secos, com temperatura alta, a produtividade foi aumentada pela irrigação, sobretudo quando a necessidade hídrica da cultura foi atendida plenamente com a reposição de $100 \%$ da ETm. Em períodos úmidos a produtividade foi influenciada pela eficiência no controle da requeima. 0 sistema Blitecast com acúmulo de 18 valores de severidade (Bli18) foi o mais eficiente para o controle da requeima da batata. A irrigação realizada em intervalos de pelo menos três dias, não exerceu influência significativa sobre a incidência e o desenvolvimento da requeima.
\end{abstract}

Palavras-chave: níveis de irrigação, Solanum tuberosum, Blitecast, produtividade

\section{Potato productivity as a function of irrigation and chemical control of Late Blight}

\begin{abstract}
The objective of this study was to determine the effect of different levels of irrigation and chemical control on yield and occurrence of Late Blight in potato CV. Asterix, at Santa M aria - RS. Two experiments were conducted at the Crop Science Department, Federal University of Santa M aria. O ne experiment was carried out during spring-summer, 2005/2006 and the second, during fall 2006. The experimental design was a complete randomized, with four replications. The Blitecast model was used to indicate the timing of spraying, by accumulating severity values (VS). Sprinkler irrigation was used corresponding to $1.0,0.75,0.50 \mathrm{ETm}$ and no irrigation. Results showed that in dry and high temperature periods, yield was affected by irrigation, mainly when crop water demand was supplied with 100\% ETm. During wet periods, yield was affected by the efficient control of Late Blight. The Blitecast model with a severity value of 18 (Bli18) was the most efficient for controlling the disease. Irrigations applied at least every three days did not affect Late Blight incidence and development.
\end{abstract}

Key words: irrigation levels, Solanum tuberosum, Blitecast, productivity

\footnotetext{
${ }^{1}$ Parte da Dissertação de Mestrado em Engenharia Agrícola do primeiro autor 2 DF/U FSM. Av. Roraima, n. 1000, Prédio 77, Cidade U niversitária, Bairro Camobi, CEP 97105-900, Santa Maria, RS. Fone: (55) 3220-8179. E-mail: edenirgrimm@yahoo.com.br; arnob.heldwein@pq.cnpq.br; sidineiradons@yahoo.com.br; ivan_maldaner@yahoo.com.br; leosane@gmail.com ${ }^{3}$ Embrapa Pecuária Sul. BR 153 Km 603, C. P. 242, Vila Industrial, CEP 96401-970 - Bagé, RS. Fone/Fax: (53) 3240-4650. E-mail: gustavo.trentin@cppsul.embrapa.br
} 


\section{INTRODUÇÃO}

Poucas são as culturas que desempenham papel tão importante como fonte de alimento para a população, como a batata, em termos de quantidade produzida e consumida (Aguiar Netto et al., 2000). Na região central do Estado do Rio Grande do Sul o cultivo da batata tem grande significância econômica e social. O cultivo é realizado, em sua maioria, por pequenos produtores, principalmente nos municípios de Silveira Martins, Ivorá, Júlio de Castilhos, Itaara, São Martinho da Serra, Restinga Seca e Santa Maria.

A cultura da batata é uma das mais exigentes em água. De maneira geral, de acordo com o ciclo e sobretudo das condições meteorológicas predominantes, essa cultura pode apresentar um consumo de água aproximado de 300 a $700 \mathrm{~mm}$ durante o período de cultivo (Garcia, 2003).

O manejo ideal da irrigação é aquele que mantém, continuamente, durante o ciclo de crescimento das plantas, condições favoráveis de umidade do solo (Bisognin, 1996) para atender à demanda de água e evitar a formação de ambiente anaeróbio no espaço do solo explorado pelas raízes. Em caso de excesso, submetendo as raízes das plantas de batata à deficiência de oxigênio, diminuindo a produção de carboidratos e provocando a morte das folhas devido às substâncias tóxicas produzidas no ambiente anaeróbico do solo (Flecha, 2004).

O efeito da irrigação por aspersão, ou por sulco na incidência de doenças, é determinado pelas condições criadas pelo sistema de irrigação, interagindo com as condições ambientais, como temperatura do ar, a umidade do ar e o tempo de molhamento da parte aérea da planta, tipo de solo, intensidade e frequência de irrigação, fatores relacionados ao manejo da cultura, variedade plantada, seu porte e espaçamento (Vale \& Zambolim, 1996).

A umidade relativa e a temperatura do ar são as variáveis meteorológicas mais importantes na infecção por Phytophthora infestans (Mont.) de Bary. A principal condição que favorece o desenvolvimento da requeima é a temperatura entre 7,2 a 26,6 ${ }^{\circ} \mathrm{C}$ (Krause et al., 1975) em período prolongado de umidade alta ou molhamento foliar. Hijmans et al. (2000), afirmam que o ciclo de vida de $P$. infestans é altamente dependente das condições ambientais, o que faz a requeima ser sensivelmente variável no espaço e no tempo. A partir desta característica é possível estudar o desenvolvimento da requeima com base no monitoramento de variáveis meteorológicas (Costa et al., 2002, Duarte et al., 2007; Trentin et al., 2009).

O molhamento da parte aérea favorece a ocorrência da requeima, que traz grandes prejuízos aos produtores de batata por ser uma doença de difícil controle. Em condições com temperatura amena e muita umidade, pode destruir uma lavoura em poucos dias (Trentin et al., 2009; Bosco et al., 2009).

Na região em que foi desenvolvido o estudo, os produtores utilizam programas fitossanitários que estabelecem um calendário semanal de aplicação de fungicidas para melhorar a eficiência do controle e prevenção de pragas e doenças (Bisognin, 1996; Bosco et al., 2009), o que é um exagero, uma vez que ao longo de um ciclo de cultivo é muito improvável que ocorra um período em que as condições ambientais sejam favoráveis aos patógenos e, portanto, nele é desnecessária a aplicação de defensivos (Batista et al., 2006). Neste sentido, são imprescindíveis pesquisas para o desenvolvimento de estratégias de manejo para a requeima com um número menor de pulverizações (Batista et al., 2006; Trentin et al., 2009).

Em função da falta de critérios para a aplicação de fungicidas, foram desenvolvidos alguns modelos para a previsão do aparecimento da requeima, por se tratar de uma doença com alto potencial destrutivo sob condições favoráveis ao seu desenvolvimento. Um dos modelos de previsão para o controle desta doença mais utilizado, é o Blitecast, desenvolvido por Krause et al. (1975), por prever o primeiro aparecimento da doença e recomendar a aplicação de fungicidas baseada no número de dias favoráveis para o desenvolvimento da doença.

$\mathrm{O}$ uso de sistemas de previsão com base nas condições ambientais apresenta algumas vantagens, como maior lucro ao produtor, decréscimo do risco de ocorrência de epidemias, redução do número de pulverizações e menor dano ao homem e ao ambiente (Wójtowicz et al., 2004; Bosco et al., 2009); no entanto, não foram encontrados trabalhos que tratam do efeito da irrigação por aspersão na incidência das doenças da cultura da batata no Brasil e seu efeito nos sistemas de previsão da requeima, razão pela qual o trabalho teve como objetivo determinar o efeito de diferentes níveis de irrigação e controle químico na produtividade e ocorrência de requeima na cultura da batata cultivar "Asterix”, em Santa Maria, RS.

\section{MATERIAL E MÉTOdos}

Realizaram-se dois experimentos na área experimental do Departamento de Fitotecnia da Universidade Federal de Santa Maria (latitude: $29^{\circ} 43^{\prime} 23^{\prime}$ ' S, longitude: 53 43' 15" W e altitude: $95 \mathrm{~m}$ ). O primeiro experimento foi realizado na primavera, no período de 21/11/2005 (emergência) a 21/02/2006 (colheita) e o segundo foi implantado no primeiro semestre de 2006 e conduzido no período de 10/04 (emergência) até 13/07/2006 (colheita).

O clima da região, conforme a classificação de Köppen, é do tipo Cfa, isto é, subtropical úmido com verões quentes e sem estação seca definida. A média de temperatura do ar para o local, nos meses de junho e julho, é de $13,8^{\circ} \mathrm{C}$, enquanto para o mês de janeiro é de $24,7^{\circ} \mathrm{C}$ e a precipitação normal anual é de $1712,4 \mathrm{~mm}$, distribuída de maneira uniforme nas quatro estações do ano (Heldwein et al., 2009).

O solo pertence à Unidade de Mapeamento São Pedro, e é classificado como Argissolo Vermelho Distrófico Arênico (EMBRAPA, 2006).

$\mathrm{Na}$ semana anterior ao plantio de cada experimento foi realizado o preparo do solo da área, seguindo-se as recomendações técnicas descritas por Bisognin (1996). Realizou-se a adubação levando-se em consideração a análise de solo e a cultura plantada na estação de cultivo, anterior à instalação do experimento na mesma área.

A cultura da batata, cultivar Asterix, foi implantada nos dois experimentos, com espaçamento entre as fileiras de plantas de 0,8 e $0,3 \mathrm{~m}$ entre as plantas nas fileiras. 
A amontoa foi realizada 18 dias após a emergência (DAE), no experimento de primavera e aos $14 \mathrm{DAE}$ no experimento de outono.

Os fungicidas utilizados nos tratamentos para o controle de doenças através do sistema Blitecast, foram mancozebe [alquilenobis (ditiocarbamato)] (Dithane NT - 3,0 $\mathrm{kg} \mathrm{ha}^{-1}$ do produto comercial), oxicloreto de cobre (Cuprogarb 350 - $4 \mathrm{~g} \mathrm{l}^{-1}$ de água do produto comercial), metiram [alquilenobis (ditiocarbamato) + piraclostrobina (estrobilurina)] (Cabrio Top $-3,0 \mathrm{~kg} \mathrm{ha}^{-1}$ do produto comercial). A aplicação dos produtos foi realizada de forma alternada com produtos de ação de contato (Dithane NT e Cuprogarb) e de ação sistêmica (Cabrio Top), observando-se o intervalo mínimo de cinco dias em virtude do efeito residual do fungicida. Utilizou-se, para sua aplicação, um pulverizador costal.

O delineamento experimental utilizado no experimento foi de blocos ao acaso com quatro repetições, sendo cada parcela composta de 4 fileiras de plantas com $5 \mathrm{~m}$ de comprimento e 3,2 $\mathrm{m}$ de largura. $\mathrm{O}$ experimento constou, no total, de 20 tratamentos, sendo avaliados quatro valores de severidade do sistema Blitecast para previsão da incidência de doenças (Bli18, Bli24, Bli30 e Test).

A avaliação da irrigação dos tratamentos foi realizada da seguinte maneira: 1,0 ETm (ETm100), 0,75 ETm (ETm75), 0,5 ETm (ETm50) e 0 ETm (testemunha). A Evapotranspiração máxima da cultura (ETm) para a batata foi calculada pela equação $\mathrm{ETm}=\mathrm{Kc}$ ETo, em que Kc é o coeficiente de cultura, obtido na literatura, conforme (Franke \& König, 1994), e ETo é a Evapotranspiração de referência, determinada pelo método de Penman (ETo). A irrigação foi realizada através de microaspersores espaçados 2,5 m x 2,5 m, para obter maior homogeneidade de distribuição de água e para que não houvesse a irrigação de parcelas vizinhas de cada tratamento. O momento da realização das irrigações e o volume de água aplicado foram baseados no cálculo diário da ETm, considerando-se as condições meteorológicas diárias. Este momento foi definido como sendo o ponto em que a ETm acumulada (ETmac) atingisse $15 \mathrm{~mm}$.

O Índice de área foliar (IAF) foi determinado por estimativa através da medida semanal do comprimento (C) e da largura (L) em cm de todas as folhas de cada planta amostrada, utilizandose uma régua com resolução de $0,1 \mathrm{~cm}$. Foram escolhidas, aleatoriamente, duas plantas por tratamento, no total de 20 plantas no experimento de 2005 e 30 plantas no experimento de 2006. As medidas foram realizadas sempre nas mesmas plantas e através delas calculou-se a área de cada folha utilizando-se a equação exponencial determinada por Streck (2006)

A incidência e a intensidade de injúria por requeima foram quantificadas conforme escala de James (1971). Não houve inoculação artificial do patógeno Phytophthora infestans (Mont.) De Bary, devido à ocorrência natural do inoculo, ao longo das duas épocas de cultivo na região. A nível de campo, a incidência e a severidade da doença, quando ocorrem é por infecção natural e se concretizam apenas quando há condições ambientais favoráveis ao patógeno o que, por exemplo, não foi constatado no experimento de primavera.

Os dados coletados foram processados através de algoritmos específicos para posterior análise estatística, como a de regressão, e quando os fatores eram qualitativos foram submetidos à análise de variância e à comparação de médias pelo teste de Tukey a $5 \%$ de probabilidade de erro.

\section{RESULTADOS E DISCUSSÃO}

Pode-se observar, na Figura 1A, que no início do experimento de 2005 ocorreram precipitações irregulares que, nos primeiros 40 DAE, totalizaram $121 \mathrm{~mm}$. No entanto, deste total 82,7 mm ocorreram aos 14 DAE e, após, não ocorreu nenhuma precipitação significativa, determinando um baixo acúmulo de valores de severidade calculados pelo sistema Blitecast. Após os 40 DAE, as precipitações na área experimental ocorreram com maior frequência até os $75 \mathrm{DAE}$ e, por isso, houve acúmulo de valores de severidade (VS) que, ao final do experimento, totalizaram 70 unidades. Este acúmulo de VS evidencia as condições meteorológicas desfavoráveis ao desenvolvimento da requeima, em função da umidade relativa do ar média diária (URam), na maior parte dos dias abaixo de $90 \%$ e a Tar relativamente alta. No trabalho conduzido por Trentin et al. (2009), houve incidência da requeima no período de primavera, ao contrário deste trabalho conduzido na primavera de 2005.

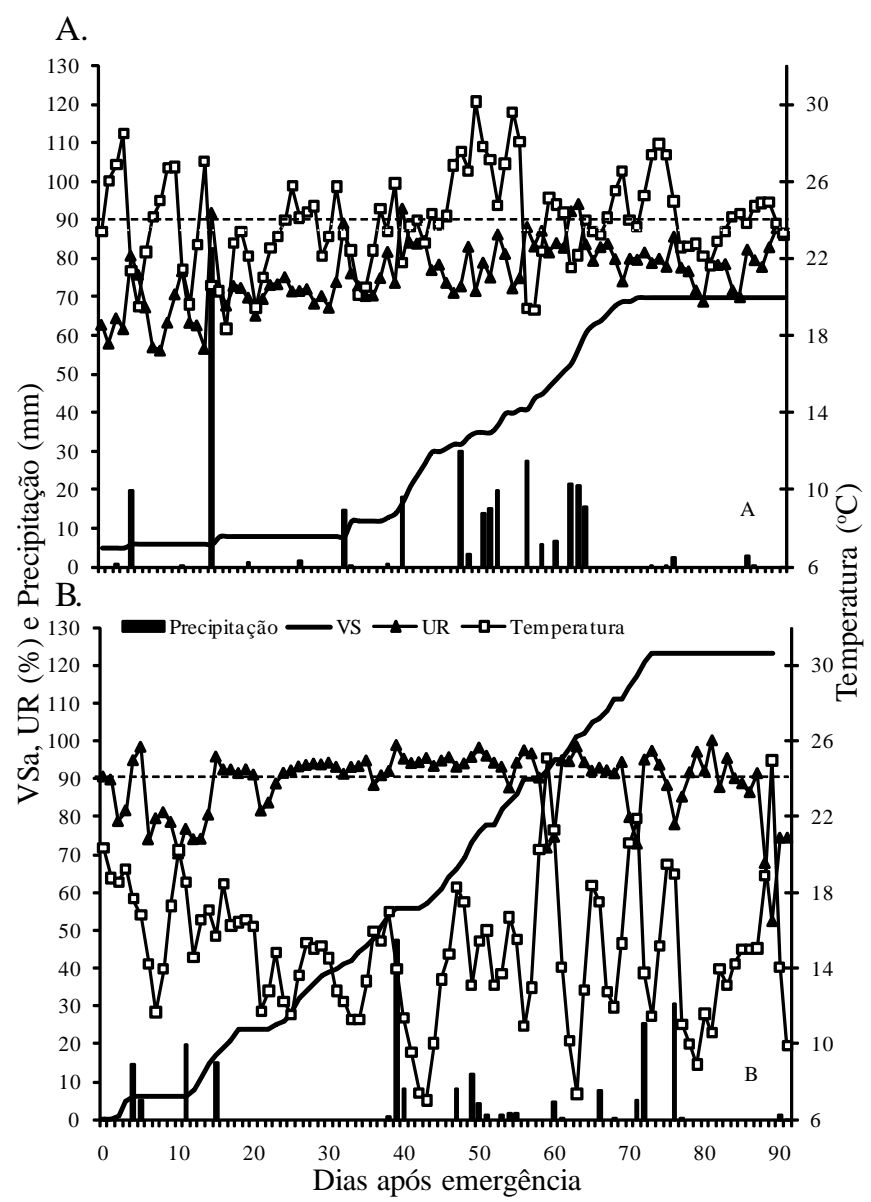

Figura 1. Valores de severidade acumulados (VSa), precipitação pluviométrica, umidade relativa do ar média diária (U Ram) e temperatura do ar média diária em dois experimentos, da primavera de 2005 (A) e do outono de 2006 (B) 
Esta condição pode estar associada à precipitação, que foi significativamente menor neste período em relação ao anterior, mantendo a superfície do solo mais seca e quente, durante o dia, o que desfavoreceu a incidência de requeima.

Na Figura 1B se verifica que no outono o acúmulo de VS foi maior, alcançando $123 \mathrm{VS}$ até o final do ciclo. Nos primeiros 15 DAE o total de precipitação foi de $54 \mathrm{~mm}$ e a URam ficou na maior parte dos dias abaixo de $90 \%$ e assim o acúmulo de VS foi relativamente pequeno. Após os $15 \mathrm{DAE}$ ocorreu um período sem precipitação, até os $40 \mathrm{DAE}$, porém nos períodos noturnos houve a formação de orvalho por períodos mais prolongados em razão da diminuição do comprimento do dia, o que promoveu um tempo maior de perda de energia da superfície do solo e das folhas durante a noite e aumentou as diferenças de temperatura do ar noturna e diurna (Streck, 2006). Assim, deu-se acúmulo de VS. Dos 40 DAE até os 75 DAE as precipitações pluviométricas somaram o total de $110 \mathrm{~mm}$ e ocorreram de forma mais regular, aumentando os períodos de horas com umidade relativa acima de $90 \%$ (HUR $\geq 90$ ) e do tempo de molhamento foliar em função da chuva e também da formação de orvalho na superfície das folhas. Desta forma, a soma dos VS foi maior. Após os 75 DAE não houve acúmulo de VS devido às condições meteorológicas desfavoráveis.

Verifica-se, na Tabela 1, que no experimento de primavera, mesmo com um volume de precipitação maior, foi necessário maior número de irrigações que no outono, devido às condições meteorológicas com temperaturas elevadas, dias mais longos, em que houve maior disponibilidade de radiação solar, o que contribuiu para maior atividade fisiológica das plantas e, consequentemente, maior consumo hídrico. A precipitação foi de aproximadamente $320 \mathrm{~mm}$ ao longo do experimento, aos quais se adicionou uma lâmina de irrigação total de $245 \mathrm{~mm}$ no tratamentoETm100. Verifica-se também que os tratamentos com irrigação apresentaram os maiores rendimentos em relação aos tratamentos sem irrigação, com variação estatística significativa

Tabela 1. Produtividade total de batata (PT), cultivar Asterix, na primavera de 2005 e no outono de 2006, em função de diferentes níveis de irrigação cal culadosa partir do acúmulo diário da ETm e aplicados em função das condições meteorológicas desfavoráveis à deriva

\begin{tabular}{|c|c|c|c|c|c|}
\hline \multirow{2}{*}{ Ano } & \multirow{2}{*}{ Tratamento } & \multirow{2}{*}{$\begin{array}{c}\text { PT } \\
\left(t h^{-1}\right)\end{array}$} & \multicolumn{2}{|c|}{ Irrigação } & \multirow{2}{*}{$\begin{array}{l}\text { Precipitação } \\
(\mathrm{mm})\end{array}$} \\
\hline & & & Número & Total $(\mathrm{mm})$ & \\
\hline $\begin{array}{c}\text { Primavera } \\
2005\end{array}$ & $\begin{array}{c}\text { ETm100 } \\
\text { ETm50 } \\
\text { ETm75 } \\
\text { Test } \\
\text { Média } \\
\text { CV (\%) }\end{array}$ & $\begin{array}{l}33,08 a \\
28,61 a b \\
26,37 a b \\
22,23 b \\
27,57 \\
16,00\end{array}$ & $\begin{array}{r}10 \\
10 \\
10 \\
0\end{array}$ & $\begin{array}{r}245,00 \\
122,50 \\
182,12 \\
0,00\end{array}$ & $\begin{array}{l}319,8 \\
319,8 \\
319,8 \\
319,8\end{array}$ \\
\hline $\begin{array}{c}\text { Outono } \\
2006\end{array}$ & $\begin{array}{c}\text { Test } \\
\text { ETm100 } \\
\text { ETm75 } \\
\text { ETm50 } \\
\text { Média } \\
\text { CV (\%) }\end{array}$ & $\begin{array}{l}26,01 \mathrm{a} \\
25,46 \mathrm{a} \\
25,23 \mathrm{a} \\
25,03 \mathrm{a} \\
25,43 \\
13,00\end{array}$ & $\begin{array}{l}0 \\
2 \\
2 \\
2\end{array}$ & $\begin{array}{r}0,00 \\
42,55 \\
21,27 \\
31,91\end{array}$ & $\begin{array}{l}214,1 \\
214,1 \\
214,1 \\
214,1\end{array}$ \\
\hline
\end{tabular}

* Valores com letras diferentes em uma mesma coluna são estatisticamente diferentes de acordo com 0 teste de Tukey $(P<0,05)$. ETm100 - Irrigação com aplicação de $100 \%$ da ETm da cultura da batata; ETm75 - Irrigação com aplicação de 75\% da ETm; ETm50 - Irrigação com aplicação de $50 \%$ da ETm; Test: Testemunha, sem irrigação entre os tratamentos ETm100 e testemunha, com diferença de produção de $10,85 \mathrm{t} \mathrm{ha}^{-1}$, sendo que esses tratamentos atingiram $33,08 \mathrm{t} \mathrm{ha}^{-1}$ e 22,23 tha ${ }^{-1}$, respectivamente. Entre os tratamentos com irrigação não houve diferença estatística.

A explicação está na disponibilidade hídrica do solo, que é um dos fatores ambientais de efeito mais visível no desenvolvimento e no crescimento da cultura da batata, influenciando diretamente na sua produtividade. A ocorrência de déficit hídrico em plantas cultivadas, como a batata, afeta o crescimento e o desenvolvimento, em todo o mundo (Santos \& Carlesso, 1998).

Na primavera, em função das elevadas temperaturas e do baixo valor de HUR $\geq 90$, o acúmulo de VS foi relativamente baixo, atingiu valor total de 70 no ciclo. Desta forma foram necessárias apenas três aplicações de fungicida, para o controle da requeima da batata no tratamento em que foram definidos 18 VS (Bli18) acumulados, como momento de aplicação. Para os tratamentos baseados no acúmulo de 24 e 30 VS (Bli24 e Bli30) foram necessárias apenas duas aplicações de fungicida (Tabela 2).

Tabela 2. Produtividade total (PT) de batata, cv Asterix, em função da aplicação de fungicidas baseada no acúmulo de 18, 24 e 30 valores de severidade de requeima (VS) (Bli18, Bli24 e Bli30), pelo sistema Blitecast, com o respectivo número de aplicações e as horas com umidade relativa acima de $90 \%$ para a primavera de 2005 e o outono de 2006

\begin{tabular}{ccccc}
\hline Ano & Tratamento & $\begin{array}{c}\text { PT } \\
\text { (t ha } \mathbf{~}^{-1} \text { ) }\end{array}$ & No Aplicações & HUR > 90 \\
Primavera & Bli18 & 27,89 a & 3 & \\
2005 & Bli24 & 27,84 a & 2 & 748,0 \\
& Bli30 & 27,34 a & 2 & \\
& Média & 27,67 & & \\
& CV (\%) & 16,00 & & \\
Outono & Bli18 & $27,97 \mathrm{a}$ & 5 & \\
2006 & Bli24 & 25,23 ab & 4 & \\
& Bli30 & $24,06 \mathrm{~b}$ & 3 & \\
& Test & $23,90 \mathrm{~b}$ & 0 & \\
& Média & 25,42 & & \\
& CV (\%) & 13,00 & & \\
\hline
\end{tabular}

* Valores com letras diferentes em uma mesma coluna são estatisticamente diferentes de acordo com 0 teste de Tukey $(P<0,05)$. Bli18 - 18 valores de severidade acumulados pelo sistema Blitecast para aplicação de fungicidas. Bli24 - 24 valores de severidade acumulados pelo sistema Blitecast para aplicação de fungicidas. Bli30 - 30 valores de severidade acumulados pelo sistema Blitecast para aplicação de fungicidas. Test: Testemunha, sem aplicação de fungicidas

O sistema Blitecast em épocas não muito favoráveis à requeima, reduz o número de aplicações de fungicidas (Katsurayama \& Bonett, 1996). Segundo Trentin (2009), dependendo das condições meteorológicas do período de cultivo é possível reduzir o número de pulverizações sem que haja perda na produção, proporcionando um incremento nos lucros do produtor, pela redução dos custos através da redução do número de aplicações, além de contribuir significativamente para a diminuição dos riscos à saúde humana e ao ambiente.

Um aspecto importante a considerar, é que a irrigação, por si só, não determina o aumento da incidência nem da severidade da requeima, mas seu efeito é determinado pelas condições criadas pelo sistema de irrigação, que interage com as condições 
ambientais, como temperatura do ar, a umidade do ar e o tempo de molhamento da parte aérea da planta, tipo de solo, intensidade e frequência de irrigação, fatores relacionados ao manejo da cultura e variedade plantada (Vale \& Zambolim, 1996).

No outono, com temperaturas mais amenas e maiores valores de HUR $\geq 90$, o acúmulo de VS foi maior e atingiu 123 pontos no final do ciclo. Desta maneira, surgiu a necessidade de maior número de aplicação de fungicidas, para o controle da requeima da batata. No tratamento Bli18 foram convenientes cinco aplicações, aos 17; 30; 40; 51 e 58 DAE. No tratamento Bli24 foram imprescindíveis quatro aplicações, enquanto no tratamento Bli30 foram feitas apenas três aplicações (Tabela 2).

Os resultados de produção obtidos no outono em função dos tratamentos com irrigação, não apresentaram diferenças estatísticas significativas em relação aos tratamentos sem a aplicação de lâminas de água. Foram apenas duas irrigações realizadas, aos 26 e aos 31 DAE. Devido às precipitações regulares, que supriram as necessidades hídricas da cultura, as diferenças nas condições de umidade do solo foram mínimas, influenciando diretamente nos resultados de produção similares obtidos. Nas duas irrigações realizadas foram aplicados 42,55 mm de água nos tratamentos com o suprimento total $(100 \%)$ da ETm. A precipitação ocorrida totalizou $214 \mathrm{~mm}$, distribuídos de forma mais homogênea em relação ao experimento de primavera. Somados a isto, a Tar média diária e a radiação solar incidente foram menores e promoveram o menor crescimento das plantas.

No experimento de outono a variação entre os tratamentos com aplicação de fungicidas foi significativa, com melhor produtividade no tratamento Bli18 que atingiu 27,97 $\mathrm{t} \mathrm{ha}^{-1}$. Os tratamentos Bli24 e Bli30, nos quais o rendimento obtido foi de 25,23 e 24,08 $\mathrm{t} \mathrm{ha}^{-1}$, respectivamente, apresentaram produtividades menores. Na comparação entre o tratamento Bli18 e os tratamentos sem a aplicação de fungicidas (Test), a diferença foi significativa $\left(4,06 \mathrm{t} \mathrm{ha}^{-1}\right)$, de maneira que no test a produção foi de 23,90 tha-1. Na comparação entre o tratamento Test, Bli24 e Bli30, não ocorreu diferença significativa mostrando que, sob irrigação, no outono, a aplicação de fungicida não pode ser retardada.

A duração do período de molhamento foliar (DPM) é mais importante do que a quantidade total de água depositada sobre as folhas, em relação ao desenvolvimento de doenças (van der Wal, 1978). Isto explica, em parte, a incidência da requeima durante o período do outono.

A análise da variância mostrou, através do teste F, que não houve interação entre a produtividade total, os tratamentos de controle da requeima através do sistema Blitecast e a aplicação de diferentes níveis de lâminas de água, tanto no experimento de primavera quanto no experimento de outono, fato possível de ser explicado pelas condições meteorológicas na região de Santa Maria. A primavera de 2005 foi o período em que ocorreu uma estiagem prolongada; no entanto, o molhamento foliar proporcionado pela irrigação não foi suficiente para favorecer a incidência de requeima; além disso, a temperatura foi elevada e com baixo valor de HUR $\geq 90$, desfavorecendo o desenvolvimento da doença.

No outono de 2006 a interação entre a irrigação por aspersão e a incidência de requeima também não foi significativa, o que pode ser explicado, sem dúvida, pelas condições meteorológicas. As temperaturas amenas e as precipitações que ocorreram, foram suficientes para suprir as carências hídricas da cultura, sendo convenientes apenas duas irrigações. Embora a incidência de requeima tenha ocorrido, ela é explicada pela Tar amena e pelo elevado valor de HUR $\geq 90$, condições essas que favoreceram a incidência e o desenvolvimento da requeima, tanto nas áreas irrigadas quanto nas áreas sem irrigação.

\section{ConClusÕES}

1. Quando a necessidade hídrica das plantas é plenamente atendida com a reposição de $100 \%$ da ETm, em períodos relativamente secos e com temperatura alta, a produtividade é significativamente influenciada pela irrigação.

2. O controle químico da requeima em períodos úmidos, incrementa a produtividade da cultura da batata.

3. Nas condições de clima da região de Santa Maria, RS, a irrigação em intervalos de pelo menos três dias em períodos secos, não exerce influência significativa sobre o desenvolvimento da Phytophthora infestans (Mont.) De Bary.

\section{AgradeCiMENTOS}

À Coordenação de Aperfeiçoamento de Pessoal de Nível Superior (CAPES) pelas Bolsas de Mestrado concedidas a Ivan Carlos Maldaner, Sidinei Zwick Radons e Gustavo Trentin, e ao Conselho Nacional de Desenvolvimento Científico e Tecnológico (CNPq) pela Bolsa de Doutorado concedida a Edenir Luis Grimm e Leosane Cristina Bosco e de Produtividade em Pesquisa, concedida à Arno Bernardo Heldwein.

\section{LITERATURA CITADA}

Aguiar Netto, A. O.; Rodrigues, J. D.; Pinho, S. Z. Análise de crescimento na cultura da batata submetida a diferentes lâminas de irrigação. Pesquisa Agropecuária Brasileira, v.35, p.901-907, 2000.

Batista, D. C.; Lima, M. A., Haddad, F.; Maffia, L. A.; Mizubuti, E. S. G. Validation of decision support systems for tomato early blight and potato late blight, under Brazilian conditions. Crop Protection, v.25, p.664- 670, 2006.

Bisognin, D. A. Recomendações técnicas para o cultivo da batata no Rio Grande do Sul e Santa Catarina. Santa Maria. 1996. 64p. Boletim Técnico.

Bosco, L. C.; Heldwein, A. B.; Lucas, D. D. P.; Trentin, G.; Grimm, E.; Loose, L. H. Sistema de previsão de ocorrência de requeima em clones de batata suscetíveis e resistentes. Ciência Rural, v.39, p.1024-1031, 2009.

Costa, R. V.; Zambolim, L.; Vale, F. X. R.; Mizubuti, E. S. G. Previsão da requeima da batateira. Fitopatologia Brasileira, v.27, p.349-354, 2002. 
Duarte, H. S. S.; Zambolim, L.; Jesus Júnior, W. C. Manejo da requeima do tomateiro industrial empregando sistema de previsão. Summa Phytopathologica, v.33, p.328-334, 2007.

EMBRAPA - Empresa Brasileira de Pesquisa Agropecuária. Centro Nacional e Pesquisa em Solos. Sistema Brasileiro de Classificação de Solos. Brasilia: Embrapa SPI; Embrapa Solos, 2006. 306p.

Flecha, P. A. N. Sensibilidade das culturas da batata (Solanum tuberosum L.) e da alface (Lactuca sativa L.) ao excesso de água no solo. ESALQ/USP. 2004. 68p. Dissertação Mestrado

Franke, A. E.; König, O. Determinação do coeficiente de cultura $(\mathrm{Kc})$ da batata (Solanum tuberosum L.) nas condições edafoclimáticas de Santa Maria, RS. Pesquisa Agropecuária Brasileira, v.29, p.625-630, 1994.

Garcia, C. J. B. Irrigação por gotejamento superficial e subsuperficial na cultura da batata (Solanum tuberosum) com dois sistemas de plantio. 2003. 67p. Botucatu: UNESP. Dissertação Mestrado

Heldwein, A. B.; Buriol, A. G.; Streck, N. A. O clima de Santa Maria. Ciência \&Ambiente, v.38, p.43-58, 2009.

Hijmans, R. J.; Forbes, G. A.; Walker; T. S. Estimating the global severity of potato late blight with GIS-linked disease forecast models. Plant Pathology, v.49, n.6, p.697$705,2000$.

James, W. C. An illustrated series of assessment keys for plant diseases. Their preparation and usage. Canadian Plant Disease Survey, v.51, p.39-65, 1971.
Katsurayama, Y.; Boneti, J. I. S. Viabilidade do uso de sistema de previsão para o controle da requeima da batateira na região de São Joaquim, SC. Fitopatologia Brasileira, v.21, p.105-109, 1996.

Krause, R. A.; Massie; L. B.; Hyre, R. A. Blitecast: A computerized forecast of potato Late Blight. Plant Disease Reporter, v.59, p.95-98, 1975.

Santos, F. R.; Carlesso, C. Déficit hídrico e os processos morfológico e fisiológico das plantas. Revista Brasileira de Engenharia Agrícola e Ambiental, v.2, p.287-294, 1998.

Streck, L. Determinação da duração do período de molhamento foliar em cultivos de batata. Santa Maria: UFSM, 2006. 107p. Tese Doutorado

Trentin, G.; Heldwein, A. B.; Streck, L.; Maass, G. F.; Radons, S. Z.; Trentin, R. Controle da requeima em batata cv. 'Asterix' como base para modelos de previsão da doença. Ciência Rural, v.39, p.393-399, 2009.

Vale, F. X. R.; Zambolim, L. Influência da temperatura e da umidade nas epidemias de doenças de plantas. In. Luz, W. C.; Fernandes, J. M.; Prestes, A. M.; Picinini, E. C. Revisão Anual de Patologia de Plantas. v.4, p.149-207, 1996.

van der Wal, A. F. Moisture as a factor in epidemiology and forecasting. In: Koslowski, T. T. (ed.). Water deficits and plant growth - Water and plant disease. New York: Academic Press, v.5, p.253-95, 1978.

Wójtowicz, A.; Lipa J. J.; Jörg, E. Economical effectiveness of Phytophthora infestans control according to decision support systems. Journal of Plant Protection Research, v.44, p.323-328, 2004. 